\title{
Probing the Merits of Different Event Parameters for the Identification of Light Charged Particles in CHIMERA CsI(Tl) Detectors With Digital Pulse Shape Analysis
}

L. Acosta, F. Amorini, R. Bassini, C. Boiano, G. Cardella, E. De Filippo, L. Grassi, C. Guazzoni, Member, IEEE, P. Guazzoni, M. Kiš, E. La Guidara, Y. Leifels, I. Lombardo, T. Minniti, A. Pagano, M. Papa, S. Pirrone, G. Politi, F. Porto, F. Riccio, F. Rizzo, P. Russotto, S. Santoro, W. Trautmann, A. Trifirò, G. Verde, P. Zambon, Student Member, IEEE, and L. Zetta

\begin{abstract}
We investigated the merits of different event parameters in the identification of Light Charged Particles (LCPs)
\end{abstract}

Manuscript received April 17, 2012; revised August 08, 2012, October 15, 2012, and December 05, 2012; accepted December 23, 2012. Date of publication January 30, 2013; date of current version February 06, 2013. This work was supported in part by the Italian Istituto Nazionale di Fisica Nucleare in the framework of the EXOCHIM experiment and in part by the European Community's Seventh Framework Programme (FP7/2007-2011) under grant agreement no 227431 . The activity was partly performed under the auspices of the MIUR (Ministero dell'Universita' e della Ricerca Scientifica) program PRIN2009, protocol 2009RLCYL8.

L. Acosta, F. Amorini, and P. Russotto are with Laboratori Nazionali de Sud-I-95123 Catania, Italy (e-mail: acosta@Ins.infn.it; amorini@Ins.infn.it; russotto@lns.infn.it).

R. Bassini, C. Boiano, P. Guazzoni, F. Riccio, and L. Zetta are with Dipartimento di Fisica dell'Università degli Studi and INFN, Sezione di Milano, I-20133 Milano, Italy (e-mail: roberto.bassini@mi.infn.it; ciro.boiano@mi.infn.it; paolo.guazzoni@mi.infn.it; filippo.riccio@mi.infn.it; luisa.zetta@mi.infn.it)

G. Cardella, E. De Filippo, A. Pagano, M. Papa, S. Pirrone, and G. Verde are with INFN, Sezione di Catania, I-95123 Catania, Italy (e-mail: Giuseppe. Cardella@ct.infn.it; enrico.DeFilippo@ct.infn.it; pagano@ct.infn.it; papa@ct. infn.it; pirrone@ct.infn.it; verde@ct.infn.it).

L. Grassi was with Dipartimento di Fisica e Astronomia, Università di Catania and INFN, Sezione di Catania, 95123 Catania, Italy. She is now with Ruđer Bošković Institute, HR-10002 Zagreb, Croatia (e-mail: laura@lnr.irb.hr)

C. Guazzoni and P. Zambon are with Politecnico di Milano-Dipartimento di Elettronica e Informazione and INFN Sezione di Milano, I-20133 Milano, Italy (e-mail: Chiara.Guazzoni@mi.infn.it).

M. Kiš is with Physikalisches Institut der Universität Heidelberg, D-69120 Heidelberg, Germany and Ruđer Bošković Institute, HR-10002 Zagreb, Croatia (e-mail: m.kis@gsi.de).

E. La Guidara is with Centro Siciliano Fisica Nucleare e Struttura della Materia, I-95123 Catania, Italy (e-mail: laguidara@Ins.infn.it).

Y. Leifels and W. Trautmann are with GSI Helmholtzzentrum für Schwerionenforschung GmbH, D-64291 Darmstadt, Germany (e-mail: Leifels@gsi.de; trautmann@gsi.de).

I. Lombardo was with Dipartimento di Fisica e Astronomia, Università di Catania and INFN, Sezione di Catania, 95123 Catania, Italy. He is now with INFN, Sezione di Napoli and Dipartimento di Fisica, Università di Napoli, I-80126, Napoli, Italy (e-mail: lombardo@na.infn.it).

T. Minniti, S. Santoro and A. Trifirò are with INFN, Gruppo Collegato di Messina and Dipartimento di Fisica, Università di Messina, I-98100 Messina, Italy (e-mail: Minniti@unime.it; ssantoro@unime.it; atrifiro@unime.it).

G. Politi is with Dipartimento di Fisica e Astronomia, Università di Catania and INFN, Sezione di Catania, 95123 Catania, Italy (e-mail: politi@ct.infn.it).

F. Porto and F. Rizzo are with Dipartimento di Fisica e Astronomia, Università degli Studi di Catania and INFN, Laboratori Nazionali del Sud (e-mail: porto@lns.infn.it; rizzo@lns.infn.it).

Color versions of one or more of the figures in this paper are available online at http://ieeexplore.ieee.org.

Digital Object Identifier 10.1109/TNS.2013.2237789 with CsI(TI) scintillators read out by photodiodes at high incident energy $(400 \mathrm{MeV} / \mathrm{u})$. This investigation is made possible by digital signal processing the output signals. As in the conventional analogue case, the digitized signals allow the discrimination of light charged particles by computing the fast and slow components. In addition other identification parameters as the rise time of the output pulses of the CsI(Tl) come out nearly for free. Aim of this paper is the investigation of novel identification plots and the probe of their merits, in particular at relativistic energies.

When the energies of the light charged particles exceed those corresponding to their ranges in $\mathrm{CsI}(\mathrm{Tl})$, the points related to the punching-through particles gather in the corresponding scatter plots giving rise to a cusp. The punch-through energies provide a complete set of data for the energy calibration of the reaction products stopped in the CsI(TI).

The obtained results suggest that at relativistic beam energies the energy vs. rise time computation provide an efficient figure for the discrimination in charge and mass of LCPs.

Index Terms-CsI(TI) scintillators, intermediate energy nuclear physics, online digital signal processing, particle identification, pulse shape analysis.

\section{INTRODUCTION}

D ETECTION and identification of Light Charged Particles (LCPs), $Z \leq 4$, are key ingredients in designing modern large area detectors aimed at studying heavy ion reactions at intermediate energies.

In nuclear physics experiments at intermediate energies the identification of charged products is implemented with Si-CsI(Tl) telescopes, as in the CHIMERA multidetector array [1]. The CHIMERA $4 \pi$-multidetector array uses detection cells made by large-area $\left(25 \mathrm{~cm}^{2}\right)$ thin silicon detectors $(300$ $\mu \mathrm{m})$ followed by Caesium Iodide Thallium activated $(0.1 \%)$ scintillators (up to $12 \mathrm{~cm}$ thick), each one coupled with a photodiode for the identification of the emitted products in mass and charge. As it is well known, the time-dependence of the light output of a CsI $(\mathrm{Tl})$ crystal, $L(t)$, is characterized - within a given energy range - by a combination of two components [2] that can be described by exponential functions with different time constants and intensities:

$$
L(t)=L_{1} \exp \left(-t / \tau_{f}\right)+L_{2} \exp \left(-t / \tau_{s}\right)
$$


where $L_{1}$ and $L_{2}$ are the light amplitudes for the fast and slow components respectively, with time constants $0.4 \mu \mathrm{s} \leq \tau_{\mathrm{f}} \leq$ $0.7 \mu \mathrm{s}$ and $\tau_{\mathrm{s}}$ up to $6 \mu \mathrm{s}$. The fast time constant depends on the mass, charge and energy of the detected particle, as well as the relative intensity $L_{1} / L_{2}$, while the slow one shows only a moderate dependence on the particle type. This dependence is the basic feature that allows particle identification by the widely used pulse shape discrimination technique.

In our previous works [3]-[5] we investigated the applicability of Digital Pulse Shape Acquisition (DPSA) methods to a typical CHIMERA detection cell and we showed the feasibility of high-resolution particle identification by means of direct digitization of the output waveforms of the CHIMERA telescope preamplifiers. For products punching through the silicon detectors, the charge identification is based on the $(\Delta \mathrm{E}, \mathrm{E})$ method and the isotopic identification of LCPs is based on the computation of the fast (F) and slow (S) components of the CsI(Tl) signals, by means of a suitable modification of the two-gate method [6]. Our previous tests [3]-[5] — carried out at intermediate energies - have proven that DPSA gives better results than standard analogue electronics, and transfers the computational burden to the on-board DSP instead of requiring dedicated analogue electronics circuits.

The aim of this paper is the investigation of novel identification plots particularly suited for particle identification at relativistic energies. The experimental data are obtained in a recent experiment at GSI where we applied digital signal processing to the waveforms acquired from the CHIMERA Si-CsI(Tl) telescopes using a ${ }^{96} \mathrm{Zr}$ beam at $400 \mathrm{MeV} / \mathrm{u}$ on a ${ }^{96} \mathrm{Zr}$ target. In particular-guided by a detailed modeling of the scintillator output waveforms - we propose two novel scatter plot representations in order to obtain LCP identification at relativistic energies. The energy of the LCPs varies in the range 50-350 $\mathrm{MeV} / \mathrm{u}$. The novel identification plots are the PulseHeight $_{\mathrm{CsI}}$, RiseTime) and the (PulseHeight ${ }_{\mathrm{CsI}}$, Slow/Fast) and we compare their merits with the conventional (Fast, Slow) representation. In this paper we also tackle the crucial problem of the energy calibration of the CsI(Tl) scintillators and we propose the exploitation of the information obtained from a Si-CsI(Tl) telescope, to carry out, in a quite straightforward way, the energy calibration for LCPs.

\section{THE EXPERIMENT}

\section{A. Experimental Setup}

The results presented here were acquired in May 2011 in the framework of the ASY-EOS experiment [7] at GSI in Darmstadt. This experiment is focused on the measurement of direct and elliptic flows of neutrons, protons, and light complex particles in reactions of isospin asymmetric neutron rich systems, as: ${ }^{197} \mathrm{Au}+{ }^{197} \mathrm{Au},{ }^{96} \mathrm{Ru}+{ }^{96} \mathrm{Ru}$ and ${ }^{96} \mathrm{Zr}+{ }^{96} \mathrm{Zr}$ at $400 \mathrm{MeV} / \mathrm{u}$. The results will help to study the asymmetry term of the Equation of State of the Nuclear Matter (EOS) [8] and provide quantitative information on the density dependence of the symmetry energy at densities larger than required for saturation.

The experimental setup consists of the Large Area Neutron Detector (LAND) [9] to measure neutron and proton squeeze-out, the ALADIN time of flight plastic wall [10] to detect forward emitted charged particles, the Washington University $\mu$-ball array [11] to identify the background reactions on non-target materials, the Krakow triple telescope array [12], and part of the CHIMERA charged particle detector [1] — four wheels (i.e. 8 detector rings) — for impact parameter determination and reaction plane reconstruction, assuring $2 \pi$ azimuthal coverage around the beam for light charged particle measurement. Of the $352 \mathrm{CsI}(\mathrm{Tl})$ scintillators installed at GSI, only 16 are equipped with $300 \mu \mathrm{m}$ thick silicon detectors in $\Delta E-E$ configuration.

The beam was guided in vacuum up to $2 \mathrm{~m}$ upstream from the target. A thin plastic foil read by two photomultiplier tubes tagged in time the beam arrival and acted as a start detector for time of flight measurement.

\section{B. Digital Pulse Shape Acquisition}

The measurements here presented have been performed by applying DPSA to sample and digitize the full pulse waveform from the Si-CsI(Tl) telescopes and the CsI(Tl) scintillators of the CHIMERA rings. The analogue electronic chain is composed of the charge preamplifiers [13], [14] (decay time constant $\tau_{\text {feed }}=60 \mu \mathrm{s}$ ) followed by antialiasing amplifiers [15] with adjustable bandwidth to fulfill the Nyquist requirement.

The digital DAQ relies on the SIS3150 VME-boards [16], each equipped with two Analog Devices ADSP-TS101S TigerSHARC Digital Signal Processors (DSP), 64 MB SDRAM memory, and two mezzanine sites. A SIS9300 Card [17], providing four 14-bit Sampling Analog to Digital Converters (SADCs) with selectable sampling frequency $(100,50$ or 25 $\mathrm{MS} / \mathrm{s}$ ) and programmable offset, is mounted on each mezzanine site.

The digitizers are directly connected through the VME-PCI Bridge to a host PC - used as supervisor [18] —located in the controlled area, near the detectors, and remotely controlled, via VNC software, by another PC, located in the acquisition room, responsible for data visualization and optionally off-line event parameter reconstruction. The digital data acquisition system shares the same trigger signal used for the main acquisition of the ASY-EOS experiment.

A detailed description of the system architecture is given in [19]. Thanks to the possibility of performing online calculations including event parameter reconstruction the data traffic on the VME bus and on the Bridge is highly reduced.

We collected signals from $32 \mathrm{CsI}(\mathrm{Tl})$ scintillators $(12 \mathrm{~cm}$ thick, 4 per ring) and also from some silicon detectors. Furthermore we sampled the trigger pulse-shape for control purposes. All the CsI $(\mathrm{Tl})$ scintillators connected to the digital DAQ present comparable results. In the present paper we show the results obtained with the telescope R5-20E located at $\theta=12.25^{\circ}$ and $\varphi=180^{\circ}$.

The preamplifier output waveforms are sampled at $50 \mathrm{MS} / \mathrm{s}$ (20 ns time intervals) and stored in the SADC memory banks in wraparound mode. In this way the SADC continuously samples and stores the values into a circular buffer. Moreover the SIS9300 board has been used in post-trigger mode, thus allowing the storage also of a part of the signal before the trigger arrival. 2048 consecutive samples are retained for each waveform. This allows baseline reconstruction exploiting about 500 samples before the pulse arrival and pulse-by-pulse compensation for long-term variations in the baseline reference levels.

Using the on-board computation, the pulse waveforms are transferred only if it is desirable to perform further off-line 


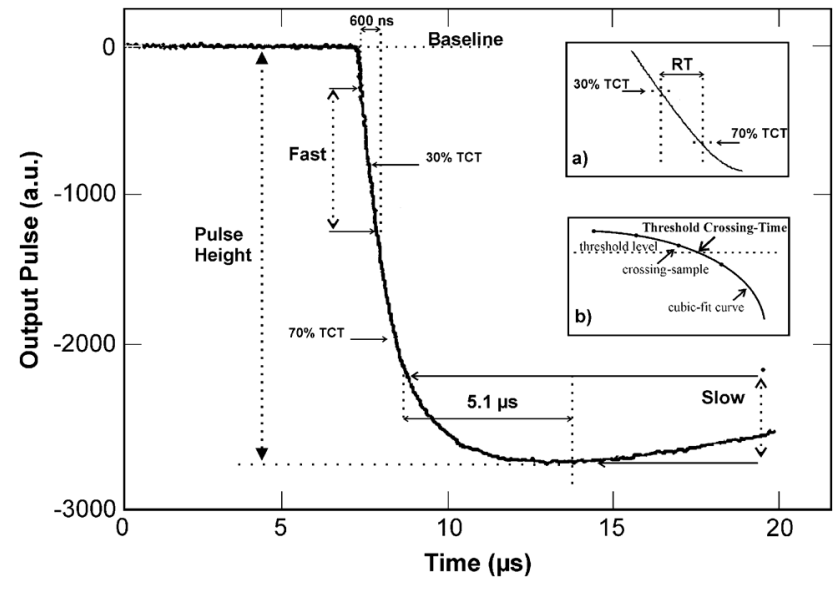

Fig. 1. Graphical representation of the criterion used to compute the event parameters. The inset a) shows the rise time calculation, while the inset b) explains the TCT algorithm.

analyses (e.g. further improvements of the algorithms or detailed studies on the acquired waveforms [20] or for diagnostic purposes). In this case a zero-suppression algorithm applied before data transferring prevents wasting transfer bandwidth. A control program on the host PC manages the SIS3150 boards through the VME-PCI bridge, downloads the pulse shapes and the data computed on-board and saves them to disk as a sequence of packets that can be read by other programs. Data collected and saved by this code are read by a custom visualizer, based on the ROOT library [21] and able to show the pulse waveforms and the event parameters computed by the DSPs, both on-line and off-line. Furthermore, an off-line analysis program allows computing additional event parameters from the stored waveforms, or the same event parameters with different settings. The algorithms used are identical to the ones used in the DSP and implemented in the same way, apart from trivial modifications due to the different system architecture.

In this work we selected the following event parameters: CsI(Tl) fast and slow pulse components, CsI(Tl) and Si pulse heights, CsI(Tl) rise time. Fig. 1 shows a typical digitized waveform. The time limits for the computation of the fast and slow components range between 0 and $600 \mathrm{~ns}$ for the fast component and between $900 \mathrm{~ns}$ and $6 \mu$ s for the slow component. All the parameters rely on a Threshold Crossing Time (TCT) algorithm, to obtain a timing of the threshold crossing more precise than the sampling time interval. All waveforms are processed with a baseline restorer algorithm and a Finite Impulse Response (FIR) filter. The processing algorithms are described in detail in [19]. Several elements influence the execution time of the algorithms. In particular, the execution time of the TCT algorithm depends on the position of the sample crossing the threshold along the pulse waveform. The execution time of the FIR filter is proportional to the number of taps in the filter kernel. Table I shows the mean execution times for several algorithms computed using the SIS3150 VME-Board and measured using the VisualDSP++ simulator [22].

Note that the TCT algorithm is used once for computing the start time (used to set the position of the fast and slow gates) and twice for computing the rise time. The measured execution time
TABLE I

EXecution Time of SEVEral Algorithms COMPUTEd Using the SIS3150 VME-BOARD

\begin{tabular}{lr}
\hline Algorithm & $\begin{array}{r}\text { Mean execution time } \\
(\mu \mathrm{s})\end{array}$ \\
\hline Baseline subtraction & 39 \\
FIR filtering (27 taps) & 164 \\
FIR filtering (87 taps) & 445 \\
Pulse Maximum search & 17 \\
Threshold crossing time & 18 \\
Rise time & 36 \\
Fast and slow components & 0.1 \\
\hline
\end{tabular}

for the processing of the $\mathrm{CsI}(\mathrm{Tl})$ pulses is, on average, about $0.6 \mathrm{~ms}$, for the 87 taps FIR filter we used for all the analyses here presented. The dominant part of the computation time is due to the FIR filter, which is unavoidable independent of the considered event parameter to provide adequate filtering for the frontend electronics series noise.

Since our main goal was the assessment of the merits of different event parameters in particle discrimination capability, we computed all the event parameters in the same acquisition runs and we did not try to benchmark the fast and slow gate method against the rise time method with respect to the computing time.

\section{MODELING}

The search for different identification plots for particle identification at relativistic energies prompted us to investigate the dependence of the pulse rise-time on the pulse amplitude and its relationship with the fast and slow components. The choice of the pulse rise-time as possible event parameter is suggested by the Pausch technique [23] performed for particles stopping in the silicon layer, since the decay constant $\tau_{\mathrm{f}}$ associated with the fast component depends [24] on the ionization density for ion charges of $Z \leq 4, \tau_{\mathrm{f}} \sim(\mathrm{dE} / d x)^{-1}=\mathrm{f}(\mathrm{E}, \mathrm{Z}, \mathrm{A})$.

Under the assumption that the scintillator light output is given by (1) and assuming the preamplifier transfer function to be the one of an ideal non-inverting integrator, the preamplifier output is given by the sum of a function due only to the fast light output and one due to the slow light output:

$$
\begin{aligned}
v_{\text {out }}(t) & =v_{\text {fast }}(t)+v_{\text {slow }}(t) \\
& =L_{1} \tau_{f}\left[1-\exp \left(-t / \tau_{f}\right)\right]+L_{2} \tau_{s}\left[1-\exp \left(-t / \tau_{s}\right)\right]
\end{aligned}
$$

where $L_{1}$ and $L_{2}$ are the light amplitudes for the fast and slow components respectively and $\tau_{f}$ and $\tau_{s}$ are the associated decay time constants. For the actual shape of the preamplifier output when the input current has the shape given by (1), see [20]. The data set used for this analysis are the ones collected at Laboratori Nazionali del Sud with a CHIMERA telescope and a 21.5 $\mathrm{MeV} / \mathrm{u}^{20} \mathrm{Ne}$ beam bombarding a ${ }^{12} \mathrm{C}$ target and deeply studied in [20] to obtain the dependence of the CsI(Tl) scintillation time constants and intensities on particle's energy, charge and mass.

Due to the transcendental dependence of the preamplifier output on time shown in (2), it is not possible to find a closed expression for the pulse rise time as a function of the light output. However, some approximations are possible that highlight the relationship between the pulse rise time, the fast and slow components and the pulse height. 


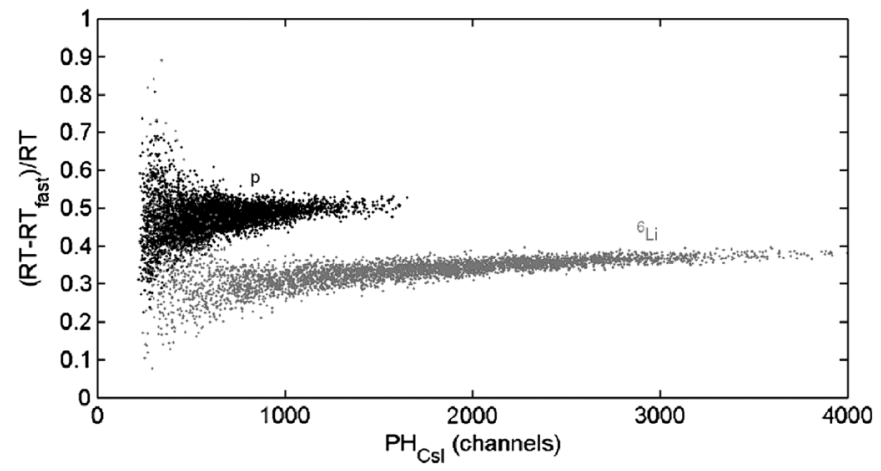

Fig. 2. Relative variation of the pulse rise time and of the rise time computed taking into account only the fast light output $\mathrm{RT}_{\text {fast }}$ as a function of the pulse amplitude for protons (black dots) and ${ }^{6} \mathrm{Li}$ ions (gray circles).

If we consider only the fast light output in (2) and compute it for the two time values defining the pulse rise time, the rise time $\left(R T_{\text {fast }}\right)$ is given by:

$$
R T_{f a s t}=\tau_{f} \log \left[\frac{(1-a)}{(1-b)}\right]
$$

where $a$ and $b$ are the two fractions of the pulse amplitude considered for the rise time computation. Taking into account the dependence of the fast time constant on the energy and on the fragment charge and mass [20], we computed the $R T_{\text {fast }}$. As illustrated in Fig. 2, in the case of the $30 \%-70 \%$ rise time (i.e. $a=0.3$ and $b=0.7$ ), for protons and ${ }^{6} \mathrm{Li}$ ions, the difference between the rise time and the so-called $R T_{\text {fast }}$ is not negligible but the two rise times can be assumed to be proportional with a coefficient nearly constant over a wide energy range.

In addition we can compute the fast and slow components as due respectively only to the fast light output $\left(\right.$ Fast $\left._{\text {fast }}\right)$ and to the slow light output $\left(S_{l o w}\right.$ slow $)$ :

$$
\begin{aligned}
\text { Fast }_{\text {fast }} & =L_{1} \tau_{f}\left[1-\exp \left(-T / \tau_{f}\right)\right] \\
\text { Slow }_{\text {slow }} & =L_{2} \tau_{s}\left[\exp \left(-T_{s, 1} / \tau_{s}\right)-\exp \left(-T_{s, 2} / \tau_{s}\right)\right]
\end{aligned}
$$

where $T$ is the upper limit of the fast gate (600 $\mathrm{ns}$ in the present case, the lower limit is $0 \mathrm{~ns})$ and $T_{s, 1}(900 \mathrm{~ns})$ and $T_{s, 2}(6 \mu \mathrm{s})$ are the lower and the upper limits, respectively, for the slow gate. Taking into account the true dependence of $L_{1}$ and $\tau_{f}$ on the energy and on the fragment charge and mass in [20], we verified that the difference between the Fast $_{\text {fast }}$ and the fast component is below $10 \%$ for all the considered energies and for $Z$ up to 3 and, in addition, Fast $_{\text {fast }}$ is proportional to the fast component. Moreover the Slow slow, computed taking into account the true dependence of $L_{2}$ and $\tau_{\mathrm{s}}$ on the energy and on the fragment charge and mass in [20] is proportional to the slow component as shown in Fig. 3.

From (3) we can express the fast time constant as a function of the rise time $\left(R T_{\text {fast }}\right)$ and therefore express the fast component (due to only the fast light output) as:

$$
\text { Fast }_{\text {fast }}=L_{1} \tau_{f}\left[1-\exp \left(-\alpha / R T_{\text {fast }}\right)\right]
$$

where $\alpha=T \log [(1-a) /(1-b)]$.

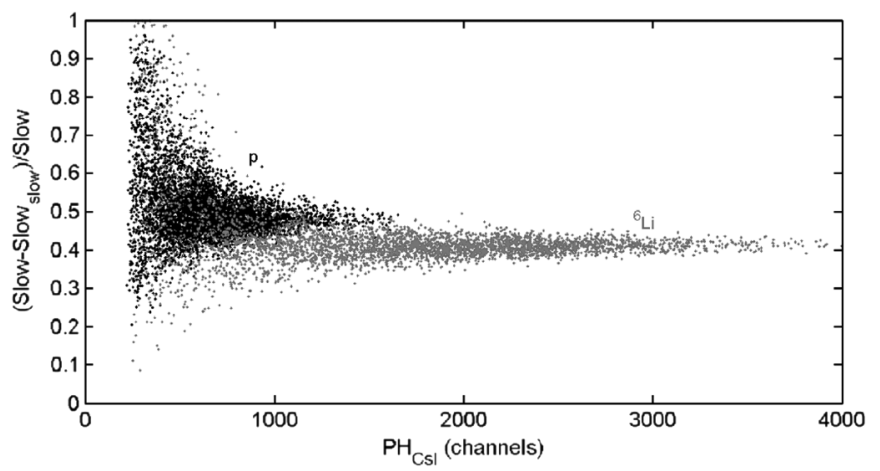

Fig. 3. Relative variation of the slow component and of the slow slow $_{\text {compo- }}$ nent as a function of the pulse amplitude for protons (black dots) and ${ }^{6} \mathrm{Li}$ ions (gray circles).

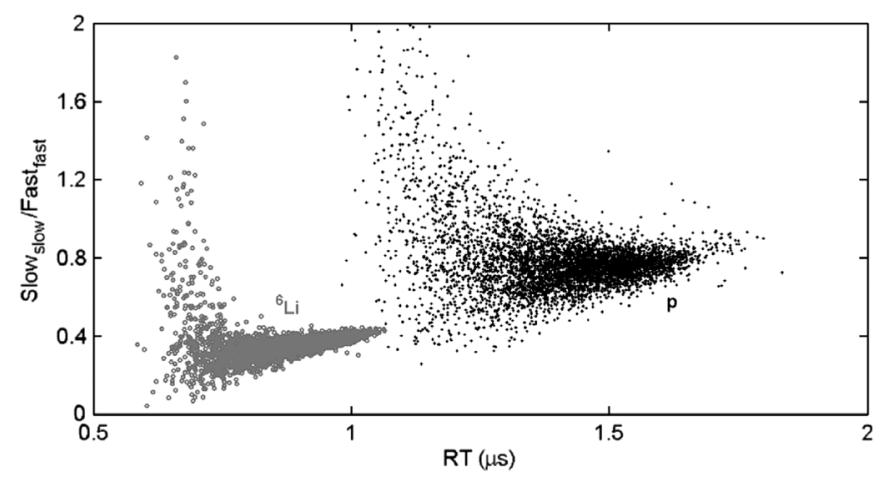

Fig. 4. Scatter plot of the Slow $_{\text {slow }} /$ Fast $_{\text {fast }}$ against the pulse rise time in the energy range of [20], for protons (black dots) and ${ }^{6} \mathrm{Li}$ ions (gray circles).

The combination of (4) and (5) leads to:

$\frac{\text { Slow }_{\text {Fast }}}{\text { Slow }_{\text {slow }}}=\frac{L_{2} \tau_{s}}{\text { Fast }_{\text {fast }}} \frac{\exp \left(-T_{s 1} / \tau_{s}\right)-\exp \left(-T_{s 2} / \tau_{s}\right)}{1-\exp \left(-\alpha / R T_{\text {fast }}\right)}$

Due to the low dependence of the slow time constant on the energy and on the fragment mass and charge, the numerator in (6) can be assumed nearly constant, in addition the ratio of the slow and fast light output intensities $\left(L_{2} \tau_{s}\right) /\left(L_{1} \tau_{f}\right)$ shows only a moderate dependence on energy and the pulse rise time and what we called $R T_{\text {fast }}$ are proportional. Moreover $\alpha$ is normally below 0.4 . Therefore the ratio of the slow and the fast components shows a nearly direct dependence on the pulse rise time since the denominator can be considered inversely proportional to the pulse rise time. This is also evident from Fig. 4 that shows the scatter plot of the Slow $_{\text {slow }} /$ Fast $_{\text {fast }}$ against the pulse rise time in the energy range of [20].

As a consequence we wanted to probe the pulse rise-time and the ratio of the Slow and Fast components against the pulse height as identification plots. As shown in Fig. 5 the pulse rise time shows a full correlation with the ratio of the Slow and Fast components. For the data at relativistic energies, presented in the present paper, the approximations considered to derive the explicit dependence of the Slow/Fast ratio on the pulse RiseTime start to fail, however the Slow/Fast ratio shows a linear dependence on the Rise-Time for ${ }^{6} \mathrm{Li}$ and for $\alpha$-particles up to $\mathrm{E}_{\alpha} \sim 500 \mathrm{MeV}$ as shown in Fig. 6, while for higher energy $\alpha$ 's and for protons we observe a clear deviation from linearity. In any case we expect a similarity of the $\left(\mathrm{PH}_{\mathrm{CsI}}, \mathrm{RT}\right)$ and $\left(\mathrm{PH}_{\mathrm{CsI}}\right.$, 


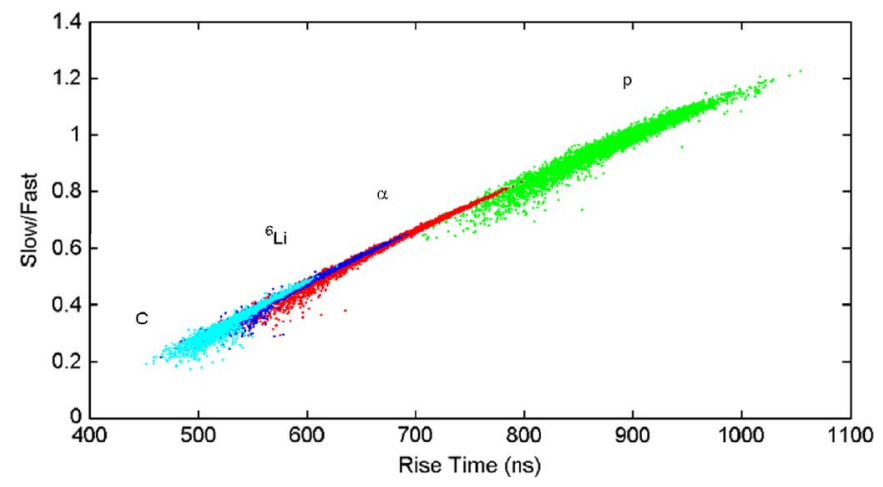

Fig. 5. (Slow/Fast, RT) scatter plot for ${ }^{20} \mathrm{Ne}+{ }^{12} \mathrm{C}$ at $21.5 \mathrm{MeV} / \mathrm{u}$ [20]. Data for different particles are shown in different colors.

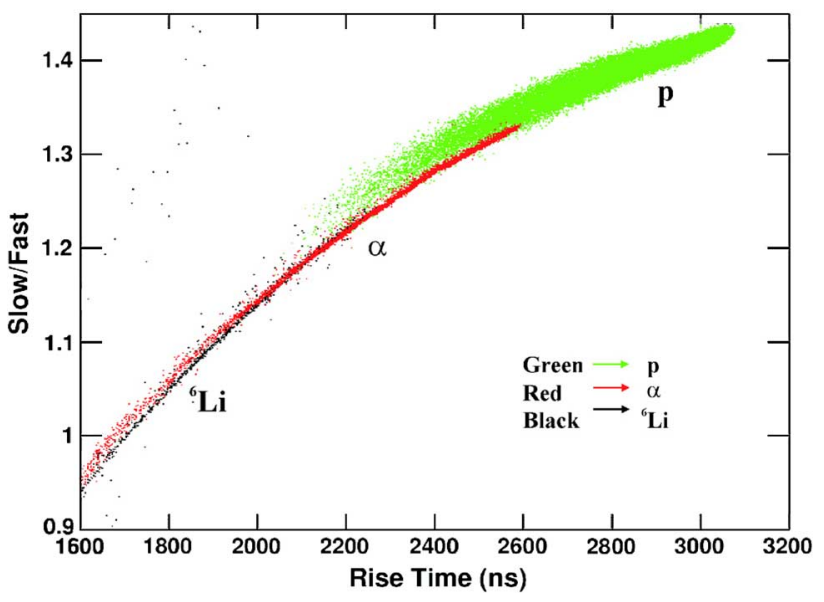

Fig. 6. (Slow/Fast, RT) scatter plot for ${ }^{96} \mathrm{Zr}+{ }^{96} \mathrm{Zr}$ at $400 \mathrm{MeV} / \mathrm{u}$. Data for different particles are shown in different colors.

Slow/Fast) scatter plots. Section IV-C will compare the merits of the newly identified identification plots.

\section{EXPERIMENTAL Procedure AND Results}

\section{A. Experimental Procedure}

The digitized waveforms have been analyzed to reconstruct the $\left(\Delta \mathrm{E}_{\mathrm{Si}}, \mathrm{E}_{\mathrm{CsI}}\right)$ and (Fast, Slow) scatter plots exploiting both the energy lost in the Silicon detector by the products crossing it and stopping in or crossing the $\mathrm{CsI}(\mathrm{Tl})$ crystal, and the fast and slow components of the decay of the emitted light output. As previously said, in order to reconstruct the $\left(\Delta \mathrm{E}_{\mathrm{Si}}, \mathrm{E}_{\mathrm{CsI}}\right)$ scatter plot we computed the maximum values of the waveforms at the output of the preamplifiers coupled with the Si detector and the photodiode reading the light output of the $\mathrm{CsI}(\mathrm{Tl})$.

The conventional (Fast, Slow) scatter plot for the data collected by the R5-20E CsI(Tl) scintillator, is shown in Fig. 7. In addition to the three clusters related to the hydrogen isotopes $(p, d, t)$ a fourth high density cluster is due to the so-called fast protons that are due to projectile multi-fragmentation.

Taking advantage of the dependence of the fast and slow components shown in Fig. 7 on the particle energy (and hence on the pulse height), we obtain a better insight into the identification of the produced fragments by scatter-plotting the height of the pulses against the ratio of the slow and fast components, as shown in Fig. 8.

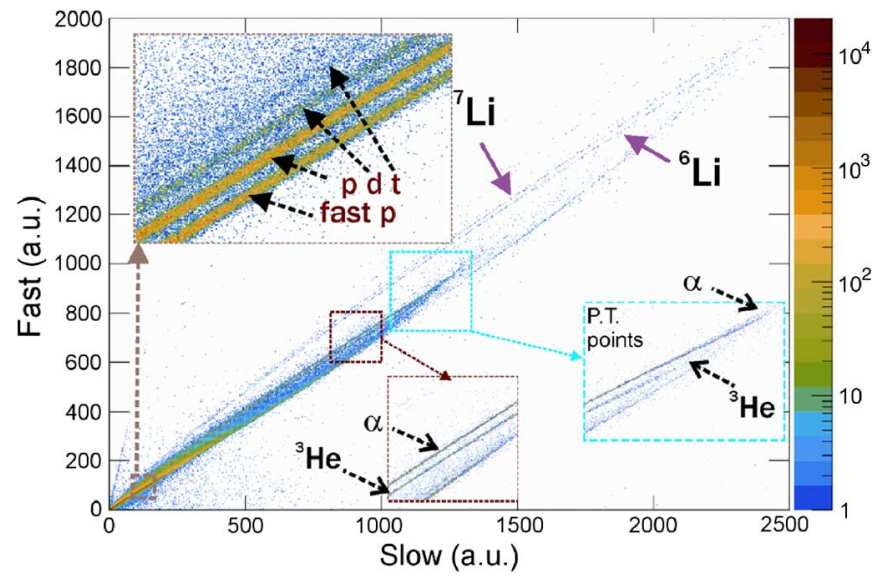

Fig. 7. (Fast, Slow) scatter plot for ${ }^{96} \mathrm{Zr}+{ }^{96} \mathrm{Zr}$ at $400 \mathrm{MeV} / \mathrm{u}$. The punchthrough points (P.T.) for ${ }^{3} \mathrm{He}$ and alphas are shown in the inset.

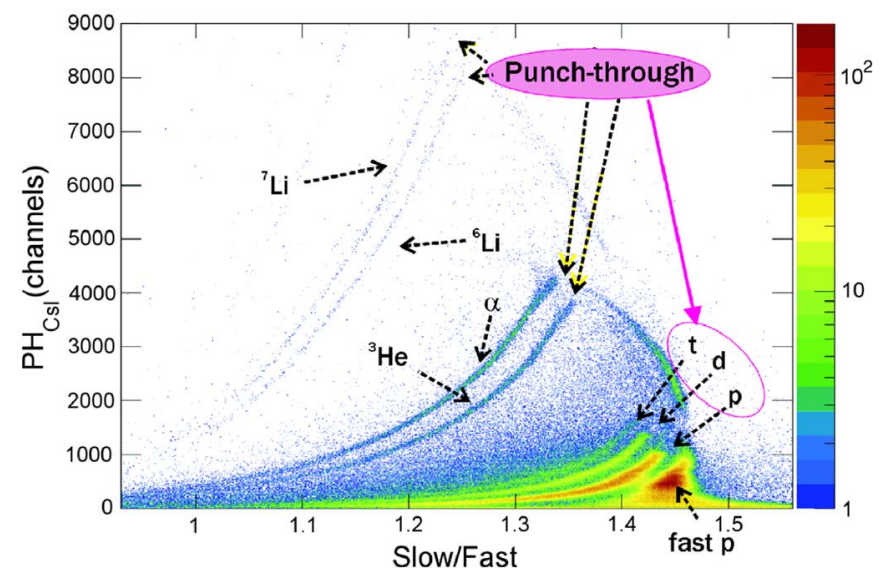

Fig. 8. $\left(\mathrm{PH}_{\mathrm{CsI}}\right.$, Slow/Fast) scatter plot for ${ }^{96} \mathrm{Zr}+{ }^{96} \mathrm{Zr}$ at $400 \mathrm{MeV} / \mathrm{u}$.

As suggested in Section III, we investigated the possibility of particle identification plotting the rise time $30 \%-70 \%$ (RT) against the pulse height $\left(\mathrm{PH}_{\mathrm{CsI}}\right)$. As a comparison, in order to illustrate the particle identification capability of the rise time computation method, Fig. 9 shows the $\left(\mathrm{PH}_{\mathrm{CsI}}, \mathrm{RT}\right)$ scatter plot obtained using the same digitized waveforms of Fig. 7. The mass and charge identification is very sharp up to $\mathrm{Z}=3$. In addition the hydrogen isotopes ( $\mathrm{p}, \mathrm{d}, \mathrm{t})$, the ${ }^{3} \mathrm{He}$ and $\alpha$ particles and the fast proton cluster are well separated.

The amount of energy deposited in the crystal depends on $\mathrm{Z}$ and $\mathrm{A}$. When the energies of the detected isotopes exceed those corresponding to their ranges in $12 \mathrm{~cm}$ thick $\mathrm{CsI}(\mathrm{Tl})$ crystals, the punch-through points appear. The gathering of the events pertaining to punching-through particles creates a cusp in the corresponding cluster in the (Fast, Slow) scatter plot. As their energies increase, the corresponding isotope lines display a backbending behavior, due to the decreased energy lost in the CsI(Tl) by the crossing isotopes. In the scatter plots of Fig. 8 and Fig. 9 the punch-through energy points and the back-bending behavior are more prominent than in the conventional (Fast,Slow) scatter plot, shown in Fig. 7.

The hint to scatter plot the pulse amplitude against the ratio of the fast and slow components derived from the marked dependence of the fast and slow components on the pulse amplitude - even more evident on the energy — found in [20]. The 


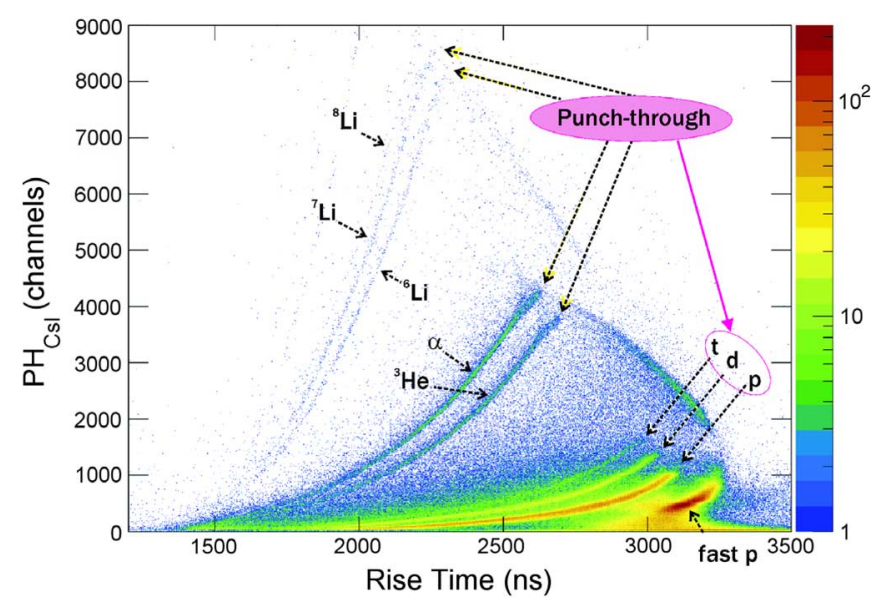

Fig. 9. $\left(\mathrm{PH}_{\mathrm{CsI}}, \mathrm{RT}\right)$ scatter plot for ${ }^{96} \mathrm{Zr}+{ }^{96} \mathrm{Zr}$ at $400 \mathrm{MeV} / \mathrm{u}$. The punchthrough points for the different identified isotopes are indicated.

better identification capability could rely on the introduction of a third variable, i.e. the pulse amplitude, that is hidden in the conventional fast-slow scatter plot. In addition due to the chosen figure of merit (see Section IV-C) that represents only a relative indicator and not an absolute one, the better identification capability does not necessarily imply that the resolution on the branches of the scatter plot is improved but that the branches separation is increased. Since the dependence of the relative intensities of the fast and slow components (with regards to the total light output intensity) is on the velocity of the impinging particle, the relative intensity of the slow (fast) component increases (decreases) with the energy per nucleon, in addition the slow (fast) component decrease (increase) with the particle charge. Due to the aforementioned dependencies, the computation of the ratio of the slow and the fast components enhances the separation of the branches pertaining to the different isotopes.

\section{B. Energy Calibration}

In order to tackle the crucial problem of the energy calibration of the CsI(Tl) scintillators, we propose a method based on the knowledge of the isotope punch-through energies in the scintillator. The cusps that correspond to punch-through energies are more prominent in the $\left(\mathrm{PH}_{\mathrm{CsI}}\right.$, Slow/Fast $)$ and $\left(\mathrm{PH}_{\mathrm{CsI}}, \mathrm{RT}\right)$ scatter plots with regard to the (Fast, Slow) scatter plot.

For each particle type, particles depositing the maximum amount of energy (and consequently giving the maximum light amplitude) in the CsI(Tl) detector are the particles having a range corresponding to the thickness of the crystal. Obviously particles with a range larger than the thickness of the CsI(Tl) crystal present an energy loss in the $\mathrm{CsI}(\mathrm{Tl})$ that decreases as the entrance energy increases. By using the SRIM code [25], we are able to compute the punch-through energy for each particle type in the CsI(Tl).

The pulse height corresponding to the punch-through energy is readily measured from the $\left(\mathrm{PH}_{\mathrm{CsI}}, \mathrm{RT}\right)$ scatter plot of Fig. 9 for each particle type. This gives us data points corresponding to the punch-through energies of $\mathrm{p}, \mathrm{d}, \mathrm{t},{ }^{3} \mathrm{He},{ }^{4} \mathrm{He},{ }^{6} \mathrm{Li}$ and ${ }^{7} \mathrm{Li}$,

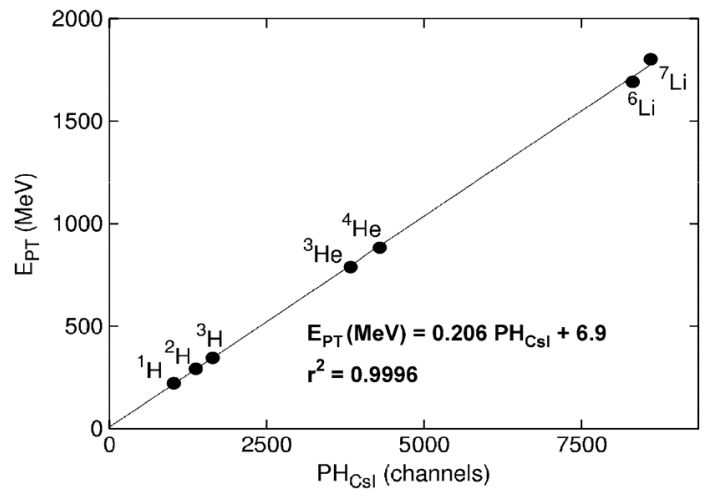

Fig. 10. Linear fit of the calculated punch-through energies in MeV to the corresponding CsI pulse heights in channels. The error bars associated with the points are smaller than the symbols used to represent the points.

which are well fit by a straight line, as shown in Fig. 10 together with the linear fit and regression coefficient $\mathrm{r}$.

A linear correspondence between pulse height and energy was not a-priori expected owing to the non-linear dependence of the light output of $\mathrm{CsI}(\mathrm{Tl})$ on the charge and energy of the impinging ion. However it has to be noted that the linear dependence is of the punch-through energies of a $12-\mathrm{cm}$ thick $\mathrm{CsI}(\mathrm{Tl})$ scintillator that lie in the energy range where the light-output tends to be linear with the energy and almost independent on the ion type.

Particles entering the CsI(Tl) detector with the punch-through energy have just left the Si detector with the same energy. Since the thickness of the Si detector is known with acceptable precision (better than 5\%) and the Si detector response is proportional to the deposited energy, independently from the particle type, it is possible to compute the energy loss in the Si detector for each particle type, by using their calculated punch-through energy values in the CsI(Tl).

To this purpose, we select the different particle clusters in the $\left(\mathrm{PH}_{\mathrm{CsI}}, \mathrm{RT}\right)$ scatter plot-as illustrated in Fig. 11 in the case of the $\alpha$-particle and deuteron clusters -, in order to obtain a $\left(\mathrm{PH}_{\mathrm{Si}}, \mathrm{PH}_{\mathrm{CsI}}\right)$ similar to a $\left(\Delta \mathrm{E}_{\mathrm{Si}}, \mathrm{E}_{\mathrm{CsI}}\right)$ scatter plot showing each particle cluster. These results are used to obtain effective $\mathrm{AZ}^{2}$ curves. The fit curves shown in the upper plots of Fig. 11 are determined by drawing a piece-wise continuous poly-line through the region of highest density. Fig. 12 shows these curves fit through the high density portion of the distributions for each particle type.

The piece-wise continuous poly-line fit through the distribution of data points can be re-parameterized into a linear function of position along the curve. From identification of the punchthrough $\mathrm{PH}_{\mathrm{CsI}}$ values in the individual particle type plots, such as shown in the upper part of Fig. 11, for alphas and deuterons, we infer knowledge of the energy of the particle as it must have leaving the Si detector.

Fig. 13 shows the calibration curve for the silicon detector. Thanks to the use of the SRIM code, we are able to relate the energy loss $\Delta \mathrm{E}_{\mathrm{Si}}$ (which is a bijective function, $\Delta E_{S i}=f\left(E_{\text {inc }}, A, Z\right)$ ) to the energy $\left(E_{\text {inc }}\right)$ of the particle hitting the silicon detector. This allows us to determine the energy deposited in the $\mathrm{CsI}(\mathrm{Tl})$ by particles with a range less than the CsI(Tl) thickness, properly using the $\mathrm{AZ}^{2}$ curves of Fig. 12, i.e. $\mathrm{E}_{\mathrm{CsI}}=\mathrm{E}_{\mathrm{inc}}-\Delta \mathrm{E}_{\mathrm{Si}}$. 

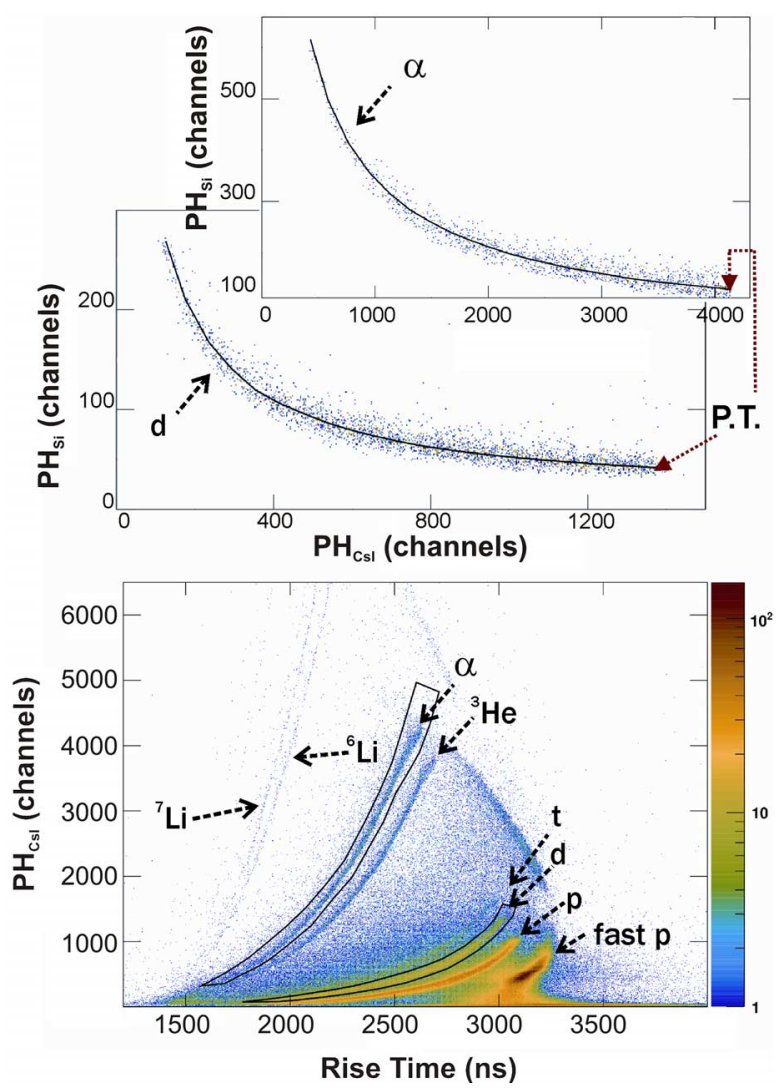

Fig. 11. Example of particle selection in the $\left(\mathrm{PH}_{\mathrm{CsI}}, \mathrm{RT}\right)$ scatter plot (bottom) and representation in individual $\left(\mathrm{PH}_{\mathrm{Si}}, \mathrm{PH}_{\mathrm{CsI}}\right)$ scatter plots, for deuterons and alphas (top). The punch-through points (P.T.) for deuterons and alphas are indicated.

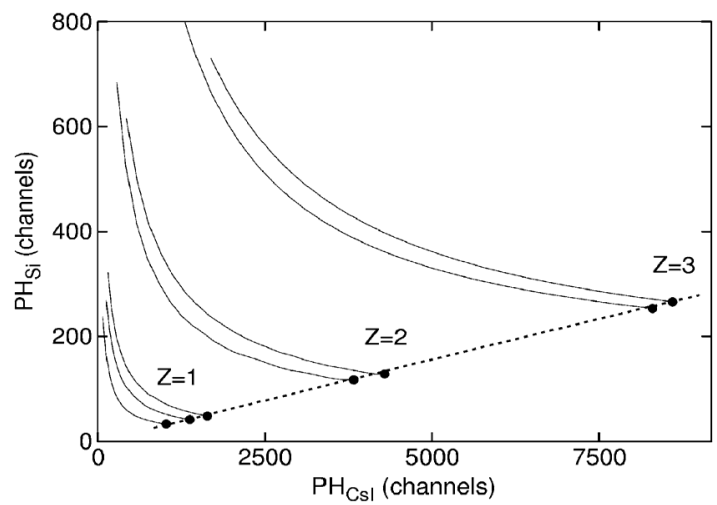

Fig. 12. $\mathrm{AZ}^{2}$ curves for $\mathrm{Z}=1,2,3$ isotopes (see text). The punch-through points are highlighted.

Fig. 14 shows the energy calibration for the $\mathrm{CsI}(\mathrm{Tl})$ scintillator obtained by linearly fitting the $\mathrm{E}_{\mathrm{CsI}}$ values obtained according to the aforementioned procedure. Even if the light output response of the $\mathrm{CsI}(\mathrm{Tl})$ scintillator does not feature a linear response [26], the non-linear dependence is most prominent at low energies. Since we are operating over a very large energy range and the minimum considered energies are above $10 \mathrm{MeV} / \mathrm{u}$ we can assume with good approximation a linear dependence of the light output on the deposited energy, as shown in Fig. 14. For sake of visibility, in Fig. 14 we shifted the $\mathrm{CsI}(\mathrm{Tl})$ energy values, measured in $\mathrm{MeV} / \mathrm{u}$, of the values (in $\mathrm{MeV} / \mathrm{u}$ ) indicated in parentheses.

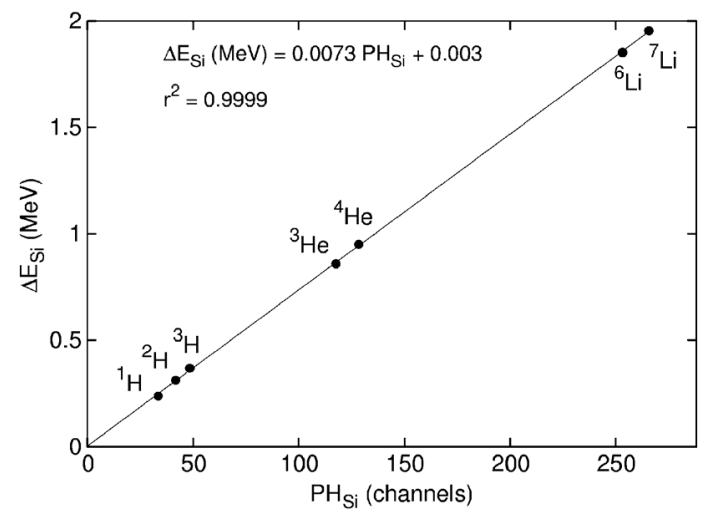

Fig. 13. Silicon detector linear energy calibration obtained by means of the indicated punch-through points energy values.

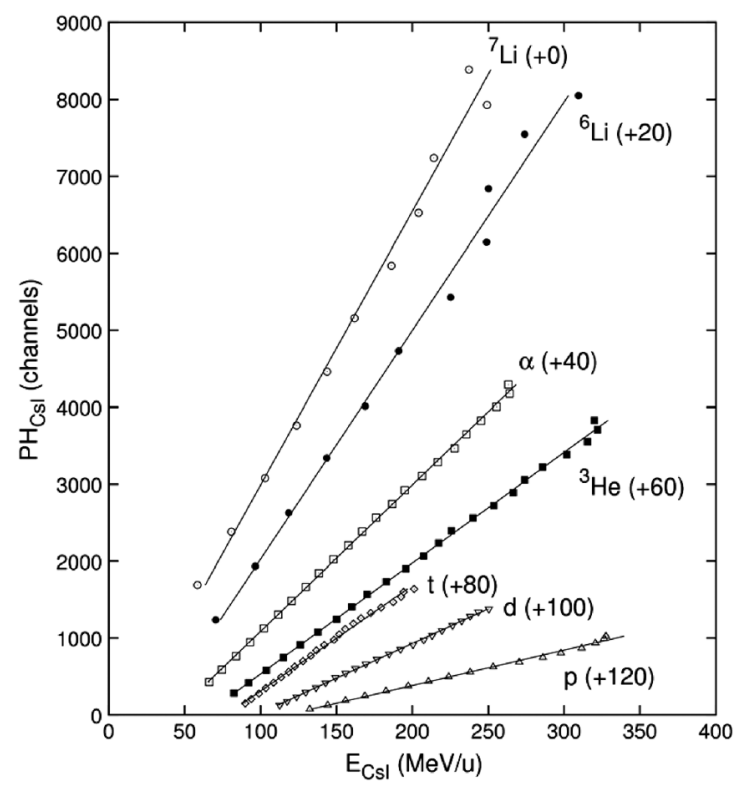

Fig. 14. CsI(Tl) scintillator energy calibration. For sake of visibility, the CsI energy values are shifted of the values indicated in parentheses.

It is important to note that this calibration method for ions stopping in the $\mathrm{CsI}(\mathrm{Tl})$ scintillators avoids time consuming experiments with monochromatic beams.

\section{Results and Analysis}

Fig. 8 and Fig. 9 clearly show the power of the (E, RT) and $(\mathrm{E}, \mathrm{S} / \mathrm{F})$ representations. In fact, the separation between protons and fast protons is very sharp, and the isotopic separation among $\mathrm{Z}=1,2,3,4$ products is evident for all energies, even towards low particle energy. Moreover the punch-through points for all the products are clearly identified. The fast protons have enough energy to punch through the $\mathrm{CsI}(\mathrm{Tl})$ crystal. The identification of this proton cluster is crucial in experiments at very high beam energies. $Z=1$ and $Z=2$ isotopes emitted in the quasi-projectile fragmentation can provide important experimental information on the EOS of the nuclear matter since their identification could contribute to the knowledge of the asymmetry term of the EOS [27].

In order to compare the LCP separation power of the three different representations of the collected data- $\left(\mathrm{PH}_{\mathrm{CsI}}\right.$, Slow/ Fast), $\left(\mathrm{PH}_{\mathrm{CsI}}, \mathrm{RT}\right)$ and (Fast, Slow) - we computed the Figure 
(a)

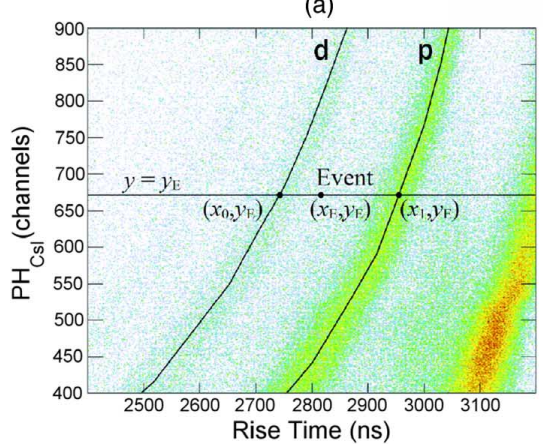

(b)

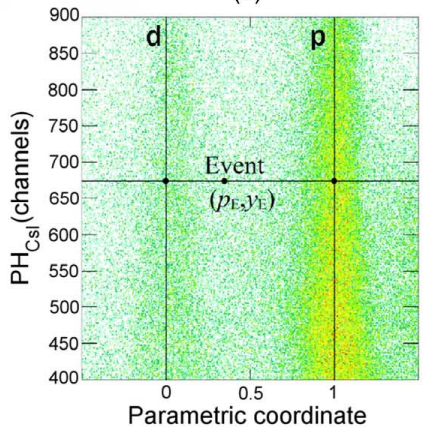

(c)

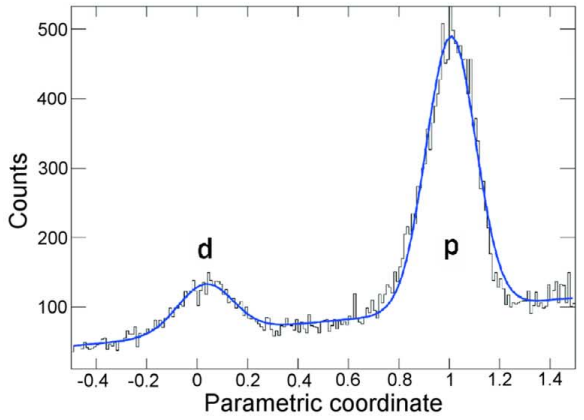

Fig. 15. The transformation used for computing the FoMs, exemplified with the pair $\mathrm{p}, \mathrm{d}$ in the $\left(\mathrm{PH}_{\mathrm{Cs}}\right.$, RT) scatter plot. In (A), the polygonals used to compute the parametric coordinate $p_{\mathrm{E}}$ for each event are shown; in (B) the representation in the transformed coordinate system $\left(\mathrm{PH}_{\mathrm{CsI}}, p_{\mathrm{F}}\right)$; in $(\mathrm{C})$ the projection of the selected pulse height interval in the transformed coordinate system; the histogram is fitted with two Gaussian and a linear background.

TABLE II

FoM VALUES FOR DiFFERENT LCP PAIRS AND REPRESENTATIONS

\begin{tabular}{cccccc}
\hline \multirow{2}{*}{ Ions } & \multicolumn{3}{c}{ Pulse Height Bin } & \multicolumn{3}{c}{ FoM (87 taps) } \\
& $\min$ & $\max$ & $\mathrm{PH}, \mathrm{S} / \mathrm{F}$ & $\mathrm{PH}, \mathrm{RT}$ & $\mathrm{F}, \mathrm{S}$ \\
\hline \multirow{6}{*}{$\mathrm{p}, \mathrm{d}$} & 300 & 400 & 1.24 & 1.17 & 1.32 \\
& 400 & 500 & 0.90 & 1.48 & 0.84 \\
& 500 & 600 & 1.41 & 1.83 & 0.97 \\
& 600 & 700 & 1.31 & 2.00 & 1.30 \\
& 700 & 800 & 1.30 & 2.15 & 1.37 \\
& 800 & 900 & 1.33 & 2.37 & 1.38 \\
\hline \multirow{6}{*}{${ }^{3} \mathrm{He}, \alpha$} & 1000 & 1500 & 2.56 & 2.46 & 2.49 \\
& 1500 & 2000 & 3.33 & 4.04 & 3.49 \\
& 2000 & 2500 & 3.70 & 4.02 & 3.30 \\
& 2500 & 3000 & 3.61 & 4.51 & 3.62 \\
& 3000 & 3500 & 3.46 & 4.07 & 3.58 \\
\hline & 2000 & 3000 & 2.15 & 1.41 & 1.89 \\
${ }^{6} \mathrm{Li}{ }^{7} \mathrm{Li}$ & 3000 & 4000 & 3.05 & 2.08 & 2.62 \\
& 4000 & 5000 & 2.78 & 2.74 & 2.82 \\
& 5000 & 6000 & 3.57 & 3.81 & 3.28 \\
& 6000 & 7000 & 3.84 & 2.80 & 2.89 \\
\hline
\end{tabular}

of Merit (FoM) [28] for various energy bins of $Z=1$ (p, d), $\mathrm{Z}=2\left({ }^{3} \mathrm{He}, \alpha\right)$ and $\mathrm{Z}=3\left({ }^{6} \mathrm{Li},{ }^{7} \mathrm{Li}\right)$ isotopes. Each energy bin contains events whose pulse height falls within a given range. The FoM is defined as the separation between two peaks, i.e. the distance between their centroids, divided by the sum of their Full Widths at Half Maximum (FWHM). The FoM is certainly one the simplest algorithm to be used for classifying particle identification because it uses only the first two moments of the distributions. Larger values of the FoM signify better identification. A value of the FoM of 0.75 corresponds to well separated identical (equal intensity and FWHM) Gaussians, with a peak-to-valley ratio of 2.0 [28].

In order to compute the FoM in the chosen scatter plot, we proceed as follows. At first, we consider each isotope cluster separately. The line of maximum density of the cluster is then approximated with a polygonal line, as shown in Fig. 15(a). We have two of these lines for each FoM to be computed. We call $\left(x_{E}, y_{E}\right)$ the coordinate of an event in the scatter plot (whatever they actually are, i.e. $\left(\mathrm{PH}_{\mathrm{CsI}}\right.$, Slow/Fast), (Fast, Slow) or $\left.\left(\mathrm{PH}_{\mathrm{CsI}}, \mathrm{RT}\right)\right)$. The two intersections of the horizontal line given by $y=y_{E}$ with the polygonals identify two points on the scatter plot, whose coordinates are given by $\left(x_{0}, y_{E}\right)$ and $\left(x_{1}, y_{E}\right)$. Along this line, we compute the parameter $p_{E}=x_{E}-x_{0} /\left(x_{1}-\right.$ $x_{0}$ ) - henceforth called parametric coordinate - which is the affine transformation constrained by the requirement that events lying on the polygonal of the first cluster have $p_{E}=0$ and events lying on the polygonal of the second cluster have $p_{E}=1$, as shown in Fig. 15(b). By using the parametric coordinate, we obtain the histogram (Fig. 15(c) ). In order to compute the FoM, the histograms are fitted with a sum of two arbitrary Gaussians representing the two peaks plus a linear background. Table II shows the values of the computed FoMs for different pairs of isotopes for all the three used identification plots and various bins for 87 taps filters. It is possible to note that only in $12.5 \%$ of the bins the usual (Fast, Slow) representation gives the best FoM.

The $\left(\mathrm{PH}_{\mathrm{CsI}}, \mathrm{Slow} / \mathrm{Fast}\right)$ representation has the best FoM in $25 \%$ of the bins, while the $\left(\mathrm{PH}_{\mathrm{CsI}}, \mathrm{RT}\right)$ representation features best results in $62.5 \%$ of the bins. A typical FoM statistical error is estimated to be $\sim 0.10$ in the worst case. We also checked the use of a narrower filter (27 taps). In this case the obtained FoM results are a little bit worse $(\sim 10 \%)$ than in the case of 87 tap filter for the $\left(\mathrm{PH}_{\mathrm{CsI}}, \mathrm{RT}\right)$ scatter plot, as expected due to the impact of the series noise of the frontend electronics. For the other two representations $\left(\mathrm{PH}_{\mathrm{CsI}}, \mathrm{S} / \mathrm{F}\right)$ and (Fast, Slow) the results are markedly worse for $\mathrm{Z}=2(\sim 40 \%)$ and $\mathrm{Z}=3$ $(\sim 30 \%)$ isotopes. For protons and deuterons with a 27 tap filter the identification is not at all possible, since at lower energies the impact of the electronic noise is even more relevant and a 27 tap filter does not provide adequate filtering for the series noise.

In order to fully probe the merits of the proposed event parameters in the identification of LCPs at relativistic energies, it is worthwhile to evaluate the false identification rates, i.e. for each pair of particles $\left(P_{1}, P_{2}\right)$ the amount of particles $P_{1}$ mistaken for particles $P_{2}$ and vice versa.

Assuming that the distribution of the particles in the considered identification histogram can be approximated with a two-Gaussian curve with centroids given by $p_{1}, p_{2}$ and FWHM $W_{1}, W_{2}$, we consider all particles in the window $\left(p_{1}-W_{1}, p_{1}+\right.$ $\left.W_{1}\right)$ as particles $P_{1}$, and all particles in the window $\left(p_{2}-\right.$ $\left.W_{2}, p_{2}+W_{2}\right)$ as particles $P_{2}$, according to [29]. This window choice assures that $98.15 \%$ of the distribution falls into it. Called $F_{1}$ the fraction of particles $P_{1}$ in the $P_{2}$ window, and $F_{2}$ the fraction of particles $P_{2}$ in the $P_{1}$ window, $F_{1}$ and $F_{2}$ depend only on the FoM and the ratio $W_{1} / W_{2}$. 


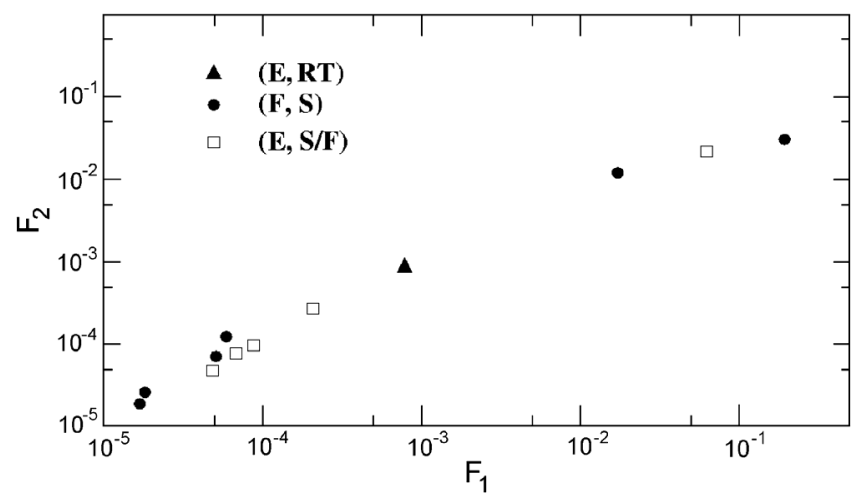

Fig. 16. Plot of the fraction of deuterons mistaken for protons $\left(F_{1}\right)$ against the fraction of protons mistaken for deuterons $\left(F_{2}\right)$ in the six energy bins of Table II (see text). The not shown values lie all below $10^{-5}$.

In our case the $W_{1} / W_{2}$ ratio is always close to 1 . With equalwidth Gaussians, the probabilities of false identification are the same for both particles and depend on the FoM only. A maximum false identification rate of $10^{-2}$ corresponds to a FoM > 1 , while a rate of $10^{-3}$ corresponds to a FoM $>1.15$.

The false identification rates for the $(\mathrm{d}, \mathrm{p})$ case are shown in Fig. 16. All other particle pairs are very well separated, with false identification rates less than $10^{-5}$.

\section{CONCLUSIONS}

In the present paper we report the results of the investigation of novel identification plots particularly suited for particle identification at relativistic energies with $\mathrm{CsI}(\mathrm{Tl})$ based detectors and digital pulse shape analysis. From the digitized waveforms we extracted the following event parameters: Fast and Slow components and rise-time that were used to compute the conventional Fast vs. Slow identification plot, the $\mathrm{PH}_{\mathrm{CsI}}$ vs. Rise-Time identification plot and the $\mathrm{PH}_{\mathrm{CsI}}$ vs. Slow/Fast identification plot.

We proposed to exploit the punch-through points for the different identified particles together with their corresponding energies - computed by means of the SRIM code - as a basis to compute the energy calibration of the $\mathrm{Si}-\mathrm{CsI}(\mathrm{Tl})$ telescope, in order to avoid the need of dedicated experiments with monochromatic beams of selected particles.

From the analysis of the collected data and the considerations on the factors of merit, we can deduce that, at least for relativistic energies, the $\left(\mathrm{PH}_{\mathrm{CsI}}, \mathrm{RT}\right)$ representation gives for the (p, d) pair of particles - the worst case - the best results in terms of false identification rate over the full considered energy range, with a false identification probability always less than $10^{-3}$. Moreover the $\left(\mathrm{PH}_{\mathrm{CsI}}, \mathrm{RT}\right)$ representation, together with the $\left(\mathrm{PH}_{\mathrm{CsI}}\right.$, Slow/Fast), allows an easier computation of the punch-through point coordinates, that allow an independent calibration in energy of the identification plots.

\section{REFERENCES}

[1] A. Pagano et al., "Fragmentation studies with the CHIMERA detector at LNS in Catania: Recent progress," Nucl. Phys. A, vol. 734, pp. 504-511, 2004
[2] F. Benrachi et al., "Investigation of the performance of CsI(Tl) for charged particle identification by pulse-shape analysis," Nucl. Instrum. Meth. Phys. Res. A, vol. 281, pp. 137-142, 1989.

[3] M. Alderighi et al., "CHIMERA data acquisition via digital sampling technique," IEEE Trans. Nucl. Sci., vol. 51, no. 4, pp. 1475-1481, Aug. 2004.

[4] M. Alderighi et al., "Charge identification in large area planar silicon detectors, using digital pulse shape acquisition," IEEE Trans. Nucl. Sci., vol. 53, no. 1, pp. 279-285, Feb. 2006.

[5] F. Amorini et al., "Digital signal processing for mass identification in a 4pi-detector, using time of flight measurement," IEEE Trans. Nucl. Sci., vol. 55, no. 2, pp. 717-722, Apr. 2008.

[6] D. Guinet, B. Chambon, B. Cheynis, A. Demeyer, D. Drain, and X. C. Hu et al., "Using the combination $\mathrm{CsI}(\mathrm{Tl})$ and photodiode for identification and energy measurement of light particles," Nucl. Instrum. Meth. Phys. Res. A, vol. 278, no. 2, pp. 614-616, 1989.

[7] [Online]. Available: http://www.ct.infn.it/asyeos2010R. C. Lemmon et al., Proposal for SIS Experiment S394 2009, unpublished

[8] W. Trautmann et al., "Differential neutron-proton squeeze-out," Prog. Part. Nucl. Phys., vol. 62, pp. 425-426, 2009.

[9] T. Blaich et al., "A large area detector for high energy neutrons," Nucl. Instrum. Meth. Phys. Res. A, vol. 314, pp. 136-154, 1992.

[10] A. Schüttauf et al., "Universality of spectator fragmentation at relativistic bombarding energies," Nucl. Phys. A, vol. 607, pp. 457-486, 1996.

[11] D. G. Sarantites et al., "The microball-design, instrumentation and response characteristics of a $4 \pi$-multidetector exit channel-selection device for spectroscopic and reaction mechanism studies with Gammasphere," Nucl. Instrum. Meth. Phys. Res. A, vol. 381, pp. 418-432, 1996.

[12] [Online]. Available: http://www.gsi.de//informationen/wti/library/scientificreport2010/index.html\#PHN-NQM-FOPIJ. Lukasik et al., Test of the Triple Telescope Prototype for the ASY-EOS Experiment

[13] C. Boiano, R. Bassini, A. Pullia, and A. Pagano, "Wide-dynamic-range fast preamplifier for pulse shape analysis of signals from high-capacitance detectors," IEEE Trans. Nucl. Sci., vol. 51, no. 5, pp. 1931-1935, Oct. 2004.

[14] C. Boiano et al., "A ultra fast hybrid charge-sensitive preamplifier for high-capacitance detectors," in Proc. Nucl. Sci. Symp. Conf. Rec., 2007, vol. 1, pp. 338-339.

[15] C. Boiano et al., "A 16-channel programmable antialiasing amplifier," in Proc. Nucl. Sci. Symp. Conf. Rec., 2010, pp. 1389-1391.

[16] "SIS 3150 VME CMC Carrier User Manual," SIS GmbH, Hamburg, Germany.

[17] “SIS 9300 ADC CMC User Manual," SIS GmbH, Hamburg, Germany.

[18] P. Guazzoni et al., "Proposed object-oriented architecture of a flexible small-scale system for digital pulse shape acquisition," IEEE Trans. Nucl. Sci., vol. 53, no. 3, pp. 886-892, Jun. 2006.

[19] F. Amorini et al., "On-board digital signal processing for $4 \pi$-detector large-area telescopes," IEEE Trans. Nucl. Sci., vol. 54, no. 1, pp. 208-213, Feb. 2007.

[20] F. Amorini et al., "Investigation of the dependence of CsI(Tl) scintillation time constants and intensities on particle's energy, charge and mass through direct fitting of digitized waveforms," IEEE Trans. Nucl. Sci., vol. 59, no. 4, pt. 3, Aug. 2012.

[21] R. Brun and F. Rademakers, "ROOT-An object oriented data analysis framework," Nucl. Instrum. Meth. Phys. Res. A, vol. 389, pp. 81-86, 1997.

[22] VisualDSP++ Version 4.0-User's Guide. Norwood, MA: Analog Devices Inc..

[23] G. Pausch, W. Bohne, and D. Hilscher, "Particle identification in solid state detectors by means of pulse-shape analysis-results of computer simulations," Nucl. Instrum. Meth. Phys. Res. A, vol. A337, pp. 573-587, 1994

[24] T. Masuda et al., "The fluorescent decay of $\mathrm{CsI}(\mathrm{Tl})$ scintillator for charged particles of different ionization density," Nucl. Instrum. Meth. Phys. Res. A, vol. 322, pp. 135-136, 1992.

[25] SRIM, The Stopping and Range of Ions in Matter [Online]. Available: http://www.srim.org

[26] E. Valtonen, J. Peltonen, and J. J. Torsti, "Response of BGO and CsI(Tl) scintillators to heavy ions," Nucl. Instrum. Meth. Phys. Res. A, vol. 286, p. $169,1990$.

[27] M. B. Tsang et al., "Constraints on the Density Dependence of the Symmetry energy," Phys. Rev. Lett., vol. 102, p. 122701 (4 pages), 2009.

[28] R. A. Winyard et al., "Pulse shape discrimination in inorganic and organic scintillators," Nucl. Instr. Meth., vol. 95, pp. 141-153, 1971

[29] Z. W. Bell, "Tests on a digital neutron-gamma pulse shape discriminator with NE213," Nucl. Instr. Meth., vol. 188, pp. 105-109, 1981. 